\title{
De la forêt naturelle aux agroforêts en Guinée forestière
}

\author{
Aboubacar Camara ${ }^{1}$ \\ Patrick Dugué ${ }^{2}$ \\ Jean-Paul Cheylan ${ }^{3}$ \\ Jean-Marie Kalms ${ }^{2}$ \\ ${ }^{1}$ Irag \\ BP 1523 \\ Conakry \\ République de Guinée \\ France \\ <aboubacar.camara@irag-guinee.org> \\ ${ }^{2}$ Cirad \\ UMR Innovation \\ TA-C 85/15 \\ 73, rue J-F Breton \\ 34398 Montpellier cedex 5 \\ France \\ <patrick.dugue@cirad.fr> \\ <jean-marie.kalms@cirad.fr> \\ ${ }^{3}$ Université d'Avignon \\ et des Pays de Vaucluse \\ 74, rue Louis Pasteur \\ 84029 Avignon cedex \\ France \\ <jean-paul.cheylan@cirad.fr>
}

\begin{abstract}
Résumé
Une démarche combinant géographie et agronomie a permis d'analyser les dynamiques spatiales des agrosystèmes forestiers en Guinée Forestière. La forte extension des associations de cultures pérennes entre elles (caféier, kolatier, cacaoyer, fruitiers) et avec une végétation forestière non plantée - dénommées " agroforêts " - a été observée dans 3 villages de la région de Kobéla. Cette dynamique spatiale peut être assimilée à un cycle de renouvellement des écosystèmes initialement dominés par la forêt. Durant la même période d'environ 25 ans, les surfaces de coteaux affectées aux cultures vivrières à base de riz pluvial venant après abattis-brûlis de recrus forestiers ont progressé modestement. Il y a eu dans ce cas une réduction de la durée de la jachère et un allongement de la durée de la culture, ce qui obère le maintien de la fertilité du sol pour ce système de culture. Par contre les agroforêts apportent des services environnementaux utiles : maintien de la biodiversité et de la fertilité du sol, contrôle du ruissellement et de l'érosion. En revanche, leur extension pourrait fragiliser la sécurité alimentaire des populations rurales en réduisant l'espace dévolu aux cultures annuelles vivrières, surtout en période de hausse des prix des produits alimentaires. Tout l'enjeu est donc de combiner à l'échelle des exploitations et des espaces villageois, les différents systèmes de culture afin de répondre durablement aux objectifs des agriculteurs et de la société.
\end{abstract}

Mots clés : agroécosystème ; agroforesterie ; développement durable ; Guinée ; système de culture.

Thèmes : systèmes agraires ; productions végétales ; forêts ; ressources naturelles et environnement ; territoire ; foncier ; politique agricole et alimentaire.

\section{Abstract \\ From natural forests to agroforests in the Guinea forest region}

A combined agronomic and geographic approach has helped to explain forest agrosystem spatio-temporal dynamics in the forest regions of Guinea. The important expansion of cropping systems associating various perennial crops (coffee, kola, cocoa, fruit trees) and native spontaneous forest species - called "agroforests"- has been observed in 3 villages of the Kobela area. This spatial dynamic can be considered as the renewing of an ecosystem dominated in the past by forest. During the same period of 25 years, the area of food crops on hills based on upland rice after slash and burn has increased slightly. In this case shortening of the fallow period has been observed simultaneously with an increase of cropping duration, leading to a soil fertility decrease trend. On the contrary, agroforests provide environmental services such as maintaining biodiversity and soil fertility, and reducing runoff and erosion. Still, they could also jeopardize food security for rural societies by reducing annual food crop areas, especially in times of an increase in food prices. The challenge is to combine these various cropping systems at the village and farm scale in order to reach both farmer and societal objectives in terms of sustainable development.

Key words: agroecosystems; agroforestry; cropping systems; Guinea; sustainable development.

Subjects: farming systems; forestry; natural resources and environment; territory; land use; agricultural and food production policy; vegetal productions. 
a plupart des diagnostics réalisés depuis un siècle en Guinée forestière sur les systèmes agricoles et ruraux mettent en avant le caractère " dégradant et non durable " des systèmes de production agricole reposant sur l'abattis-brûlis des recrus forestiers. L'imminence d'une crise écologique et alimentaire est constamment proclamée: déforestation accélérée, érosion des sols, perte de biodiversité, pénurie vivrière, etc. (Brasseur, 1956; Rossi, 1993). Ces constats alarmistes ont justifié plusieurs programmes de développement visant la protection de l'environnement par le classement de forêts et l'aménagement des bas-fonds pour limiter la défriche-brûlis des coteaux. Si le classement des forêts a été relativement efficace, les techniques d'intensification agricole ont été peu adoptées par les agriculteurs. Pour dépasser ces conclusions générales, des analyses ont été entreprises pour affiner la compréhension des changements en cours dans cette province de Guinée forestière (Delarue, 2007). Présentant des travaux réalisés dans la Communauté rurale de développement (CRD) de Kobéla (figure 1), cet article s'inscrit dans cet objectif. Nous montrerons l'évolution spatio-temporelle des systèmes de mise en valeur dans cette zone écologique de forêt humide et discuterons de leur durabilité.

\section{Matériel et méthode}

\section{Contexte}

Notre zone d'étude, la CRD de Kobéla (37 hab/ $\mathrm{km}^{2}$ en moyenne en 2005), est représentative de l'écosystème forestier de Guinée forestière. Ses paysages sont caractérisés par une succession de collines ferralitiques et de pénéplaines, drainées par des cours d'eau faiblement encaissés dans des bas-fonds hydromorphes. Le climat de type subéquatorial humide, avec 9 mois de pluie (2 000-2 $500 \mathrm{~mm} / \mathrm{an}$ ), permet une longue période végétative (280 jours par an) favorable au développement d'une agriculture très diversifiée incluant plantes annuelles et pérennes.

Dans cette région, les agrosystèmes vivriers sont essentiellement basés sur l'agriculture itinérante d'abattis-brûlis produisant principalement du riz pluvial, base de l'alimentation des populations locales (Porteres, 1966). Jusqu'à la moitié du $\mathrm{xx}^{\mathrm{e}}$ siècle, ce système de mise en valeur consistait typiquement en une période de culture de 2 à 3 ans suivie d'une jachère d'une à plusieurs décennies pour la régénération du couvert végétal, permettant l'entretien la fertilité des sols. Cet agrosystème est resté en équilibre avec les ressources naturelles mobilisées car la faible densité de population, en général inférieure à $10 \mathrm{hab} / \mathrm{km}^{2}$, induisait la mise en culture annuelle d'une petite proportion du territoire. Aujourd'hui, cet équilibre est devenu plus précaire (Rossi, 1993) car la densité de population a augmenté, atteignant plus de $50 \mathrm{hab} / \mathrm{km}^{2}$ dans certaines zones rurales (Camara, 2007). La jachère n'est plus assez longue (inférieure à 10 ans) pour permettre la régénération de la forêt secondaire et de la fertilité du sol. En dehors des aires protégées, les forêts reliques ne couvrent plus que de petites superficies. On a donc affaire à des systèmes agraires postforestiers.

Cet écosystème donne lieu à trois systèmes de mise en valeur agricole :

- le système vivrier de coteaux sur défriche-brûlis fondé sur la riziculture pluviale associée aux cultures alimentaires secondaires (gombo, piment, oseille, taro...) en rotation avec l'arachide et le manioc. Ce système est associé à des peuplements de palmiers à huile subspontanés (Elaeis dura) de densité variable (Madelaine et al., 2008) ;

- les systèmes agroforestiers complexes dénommés par la suite "agroforêts ", sont constitués de cultures pérennes comprenant une spéculation principale (café ou cacao) associée à des cultures pérennes secondaires (kolatier, fruitiers) et sont conduits sous un couvert forestier de composition variée

- le système de culture de bas-fond constitue le domaine de la riziculture inondée. Il associe à la riziculture de saison des pluies un peuplement résiduel de palmier raphia (Raphia ruffia) et parfois en rotation, des cultures maraîchères de saison sèche.

\section{Analyse géographique et agronomique des pratiques paysannes}

L'analyse géographique du territoire permet d'analyser la distribution spatiale et les dynamiques des systèmes de mise en valeur. Elle s'appuie sur une cartographie diachronique d'occupation des sols de trois villages de la CRD (Nienh, Maouon, Konipara) sur un pas de temps d'une génération humaine qui a mobilisé des photo- mosaïques au 1/50 000 de 1979 et une image satellite SPOT 5 à 2,5 m de résolution de 2003. Du fait de l'exiguité des bas-fonds dans cette petite région et de leur couverture dans le passé par une végétation dense à base de palmier raphia, il n'a pas été possible de les cartographier avec précision et d'analyser la dynamique de leur mise en valeur entre 1979 et 2003. L'approche géographique est complétée par une analyse des pratiques paysannes fondée sur des enquêtes réalisées auprès des 650 exploitations agricoles des trois villages et des entretiens avec des personnes ressources (autorités villageoises, responsables paysans). Cette méthode s'inspirant de la géoagronomie de Deffontaines (1998) permet d'identifier les évolutions des systèmes de production et des paysages ruraux.

\section{Résultats : dynamiques spatiales et évolution des systèmes de mise en valeur}

Les cartes d'occupation du sol de 1979 et 2003 (figure 2) rendent compte de trois processus de "mise en valeur " de cet écosystème forestier.

\section{Disparition des forêts " naturelles "}

La disparition de la forêt naturelle, est sans doute l'évolution la plus spectaculaire dans cette région. À Nienh, la forêt qui " bouchait l'horizon et faisait disparầtre presque complètement l'idée de relief "(Brasseur, 1956), avait déjà complètement disparu en 1979. À Konipara, elle a également totalement disparu entre 1979 et 2003. À Maouon, $20 \%$ de la surface en forêt présente en 1979 subsistent encore en 2003, grâce au reclassement du Mont Yono en aire protégée (figure 2). Tant qu'il y a une portion de forêt accessible (pas trop pentue, non classée) l'agriculture pionnière sur abattis-brûlis continue à se développer. La menace de la disparition de la forêt naturelle et de la biodiversité qu'elle renferme est bien une réalité qui justifie des mesures de protection surtout pour les espaces fragiles: massifs montagneux, forêts galeries, têtes de source. 

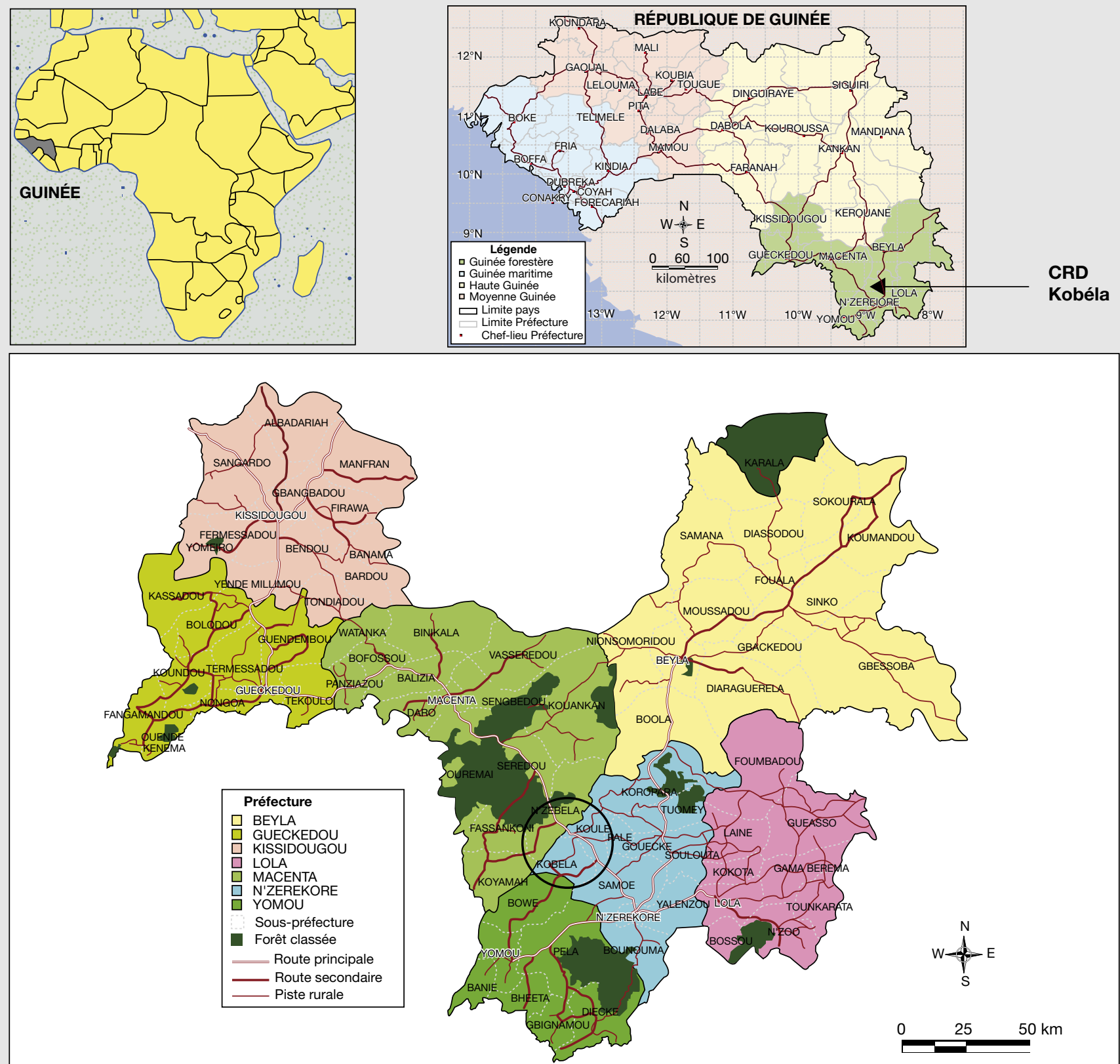

Figure 1. Localisation de la CRD de Kobéla en Guinée forestière.

Figure 1. Situation of Kobela CRD in the forest regions of Guinea.

\section{Diversité d'évolutions du système vivrier de coteaux sur abattis-brûlis}

En 2003, 70 à $80 \%$ de la surface totale des territoires villageois étudiés sont couverts par le système de culture vivrier à base de riz pluvial et jachère, sous palmiers subs- pontanés (tableau 1). Il constitue encore aujourd'hui, la principale forme d'occupation de l'espace dans cette région. Mais ce système est en pleine mutation, confronté actuellement aux pressions foncières liées à l'accroissement des besoins alimentaires locaux et urbains et à l'extension des agroforêts. Ces facteurs ont entraîné une demande importante en terre cultivée et donc le raccourcissement du temps de jachère, de 10-20 ans il y a trois décennies à 4-5 ans aujourd'hui (figure 3).

Le ratio [surface agricole utile pour les vivriers d'un territoire/surface réellement emblavée en vivriers annuels l'année i], soit $\mathrm{R}=\mathrm{SAUvi} / \mathrm{SCvi}$, permet d'exprimer l'intensité de la pression sur le foncier cultivable. À Nienh, ce ratio est passé de 


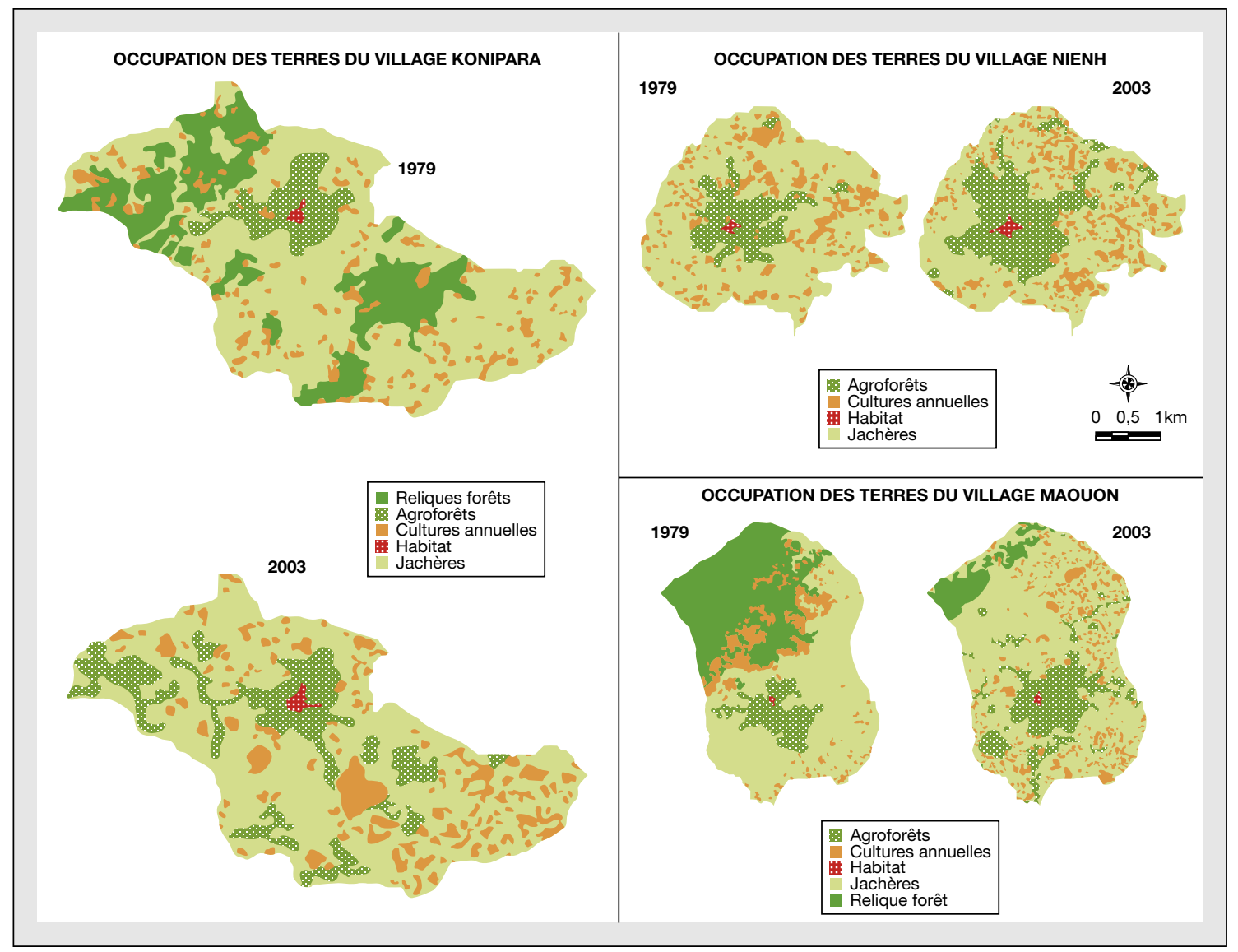

Figure 2. Évolution de l'occupation du sol dans les trois villages d'étude.

Figure 2. Land use change in the three villages.

5,2 à 4,8 entre 1979 et 2003. Les surfaces cultivées sont restées presque identiques. Cela s'explique par l'accroissement de la durée de culture (passage de 2 à 3 ans, voire 4 ans) et par une augmentation modérée $(36 \%)$ de la population en un demi-siècle : de 1142 habitants en 1955 (Brasseur, 1956) à 1773 habitants en 2005 (Camara, 2007).

À Maouon, le ratio R est passé de 9,1 à 7,4. Cette faible diminution est due à l'importance de la relique de forêt du Mont Yono, déclassée au cours des années 1970, puis reclassée en 2003. Pendant le déclassement, un front pionnier agricole s'y était installé, et $80 \%$ de la forêt ont été transformés en cultures vivrières-jachère (tableau 2). Si cette forêt classée est effectivement épargnée par de nouveaux défrichements, la pression sur la terre agricole va augmenter $(\mathrm{R}=4,3)$ bien que la surface cultivée en vivriers n'ait progressé que de $10 \%$ entre 1979 et 2003. A Konipara, le ratio $\mathrm{R}$ a évolué plus rapidement, passant de 10,3 à 5,3. Cela s'explique par une augmentation de $66 \%$ des surfaces de cultures vivrières, favorisée par le front en colonisation sur les reliques de forêt, dont $70 \%$ ont été transformées en cultures vivrières et jachère. Comme il n'y a plus de forêt à défricher, l'augmentation des surfaces cultivées ne peut désormais se faire qu'au détriment des jachères, dont la durée diminue proportionnellement. Cette situation correspond à celle qui est observée aujourd'hui à Nienh.

Cette diversité des dynamiques agricoles se traduit par des rythmes différenciés de réduction du temps de jachère, essentiellement en fonction de la disponibilité en forêt. Mais la tendance à une réduction de la durée de jachère est commune aux trois sites pour atteindre dans la majorité des cas 4 à 5 ans (figure 3). Les emblavures en cultures vivrières annuelles sur les coteaux n'ont pratiquement pas progressé à Nienh et Maouon entre 1979 et 2003, ce qui laisse supposer non seulement que la population n'a pas trop augmenté mais aussi que les rendements n'ont pas beaucoup régressé malgré la réduction de la durée de jachère. Il est aussi possible que les chefs de ménage aient trouvé d'autres solutions pour assurer la sécurité alimentaire de leur famille : les zones de bas-fonds ont toutes été mises en culture, les agroforêts pourraient fournir des revenus suffisants pour assurer entre autres, l'achat de riz, nourriture de base dans cette région.

\section{Une forte extension des agroforêts}

La diversité de composition des agroforêts est remarquable (Camara, 2007). Soixante-six pour cent des parcelles d'agroforêts comprennent encore du caféier mais ce dernier est presque toujours associé à une ou plusieurs autres cultures pérennes. Ce système d'association à base de caféier demande peu d'investissement au départ et procure un revenu régulier apprécié. La caféiculture en culture pure sous ombrage de grands arbres, ne représente que $3 \%$ des agroforêts malgré les interventions en sa faveur 
de projets de développement (projets de relance de la culture caféière) pendant au moins une décennie. Les associations à base de cacaoyer (26\% des agroforêts) et de bananier (11\%) sont issues de l'implantation d'agroforêts sur les sols profonds de bas de pente. Les associations à base d'arbres fruitiers (oranger, manguier, citronnier, goyavier) ne représentent que $3 \%$ des agroforêts. Les palmiers hybrides plantés depuis environ 5 ans, correspondent à seulement $4 \%$ des parcelles agroforestières. L'huile ainsi produite est d'ailleurs moins appréciée par les consommateurs que celle fournie par les palmiers dura subspontanés.

L'extension de ces différents types d'agroforêts est la principale dynamique observée aujourd'hui. Leur surface a plus que doublé à Konipara (169\%) et Maouon (114\%). Cette augmentation n'est que de $69 \%$ à Nienh, village ayant déjà connu un développement plus ancien. Deux principaux processus sont à l'origine de l'extension des agroforêts (tableau 1):

- la transformation des reliques de forêt en agroforêts est logique car ces espaces fournissent après un défrichement raisonné des conditions écologiques favorables au développement des cultures pérennes (ombrage, humidité, fertilité). Ce processus est classique en Afrique tropicale humide, il a été observé en Côte d'Ivoire dans les années 1980 et 1990 par Ruf (1995) et qualifié d'exploitation de la rente forêt. Il est particulièrement remarquable à Konipara où il correspond à $50 \%$ des extensions des agroforêts ;
- la transformation des espaces vivriers de coteaux (cultures annuelles et jachère) en agroforêts est encore plus remarquable. Ce processus représente $89 \%$ des extensions des agroforêts à Maouon, $100 \%$ à Nienh et $40 \%$ à Konipara. Cela correspond le plus souvent à l'installation de cultures pérennes après une ou deux années de riz pluvial. Les plants sont installés en association avec des cultures résiduelles telles que le manioc, le bananier et l'ananas. Plus rarement, quelques arbres peuvent être plantés autour de l'abri construit dans la parcelle vivrière afin d'y constituer un jardin de case arboré qui se transforme progressivement en agroforêt par plantation centrifuge d'arbres divers.

La distribution spatiale des agroforêts par rapport au centre du village et leur âge

\section{Tableau 1. Évolution de l'occupation du sol des trois villages de 1979 à 2003.}

Table 1. Land use change in the three villages from 1979 to 2003.

\begin{tabular}{|c|c|c|c|c|c|c|}
\hline \multicolumn{7}{|c|}{ Village Nienh } \\
\hline Unités d'occupation & $\begin{array}{c}\text { Surface } \\
1979 \text { (ha) }\end{array}$ & $(\%)$ & $\begin{array}{c}\text { Surface } \\
2003 \text { (ha) }\end{array}$ & $(\%)$ & $\begin{array}{c}\text { Évolution } \\
\text { (ha) } \\
\text { 1979-2003 }\end{array}$ & $\begin{array}{c}(\%) \\
1979-203\end{array}$ \\
\hline Habitat & 12 & 0,4 & 24 & 0,7 & 12 & 103 \\
\hline Agroforêt & 513 & 15,6 & 868 & 26,3 & 354 & 69 \\
\hline Cultures vivrières & 526 & 16 & 491 & 15 & -35 & -7 \\
\hline Jachère & 2217 & 68 & 1886 & 58 & -331 & -15 \\
\hline Relique forêt & 0 & 0 & 0 & 0 & 0 & 0 \\
\hline Total & 3268 & 100 & 3267,92 & 100 & & \\
\hline \multicolumn{7}{|c|}{ Village Maouon } \\
\hline Unités d'occupation & $\begin{array}{c}\text { Surface } \\
1979 \text { (ha) }\end{array}$ & $(\%)$ & $\begin{array}{c}\text { Surface } \\
2003 \text { (ha) }\end{array}$ & $(\%)$ & $\begin{array}{c}\text { Évolution } \\
\text { (ha) } \\
\text { 1979-2003 }\end{array}$ & $\begin{array}{c}(\%) \\
1979-203\end{array}$ \\
\hline Habitat & 6 & 0,1 & 12 & 0,2 & 6 & 100 \\
\hline Agroforêt & 626 & 7,9 & 1340 & 17,8 & 714 & 114 \\
\hline Cultures vivrières & 751 & 10 & 823 & 11 & 73 & 10 \\
\hline Jachère & 3606 & 48 & 4798 & 64 & 1192 & 33 \\
\hline Relique forêt & 2475 & 33 & 490 & 6 & -1985 & -80 \\
\hline Total & 7465 & 100 & 7464 & 100 & & \\
\hline \multicolumn{7}{|c|}{ Village Konipara } \\
\hline Unités d'occupation & $\begin{array}{c}\text { Surface } \\
1979 \text { (ha) }\end{array}$ & $(\%)$ & $\begin{array}{l}\text { Surface } \\
2003 \text { (ha) }\end{array}$ & $(\%)$ & $\begin{array}{c}\text { Évolution } \\
\text { (ha) } \\
\text { 1979-2003 }\end{array}$ & $\begin{array}{c}(\%) \\
1979-2003\end{array}$ \\
\hline Habitat & 9 & 0,3 & 17 & 0,5 & 8 & 85 \\
\hline Agroforêts & 254 & 7 & 684 & 19,5 & 430 & 169 \\
\hline Cultures vivrières & 311 & 8,7 & 519 & 15 & 208 & 67 \\
\hline Jachères & 2070 & 59 & 2265 & 65 & 195 & 9 \\
\hline Relique forêt & 840 & 24 & 0 & 0 & -840 & -100 \\
\hline Total & 3484 & 100 & 3484 & 100 & & \\
\hline
\end{tabular}




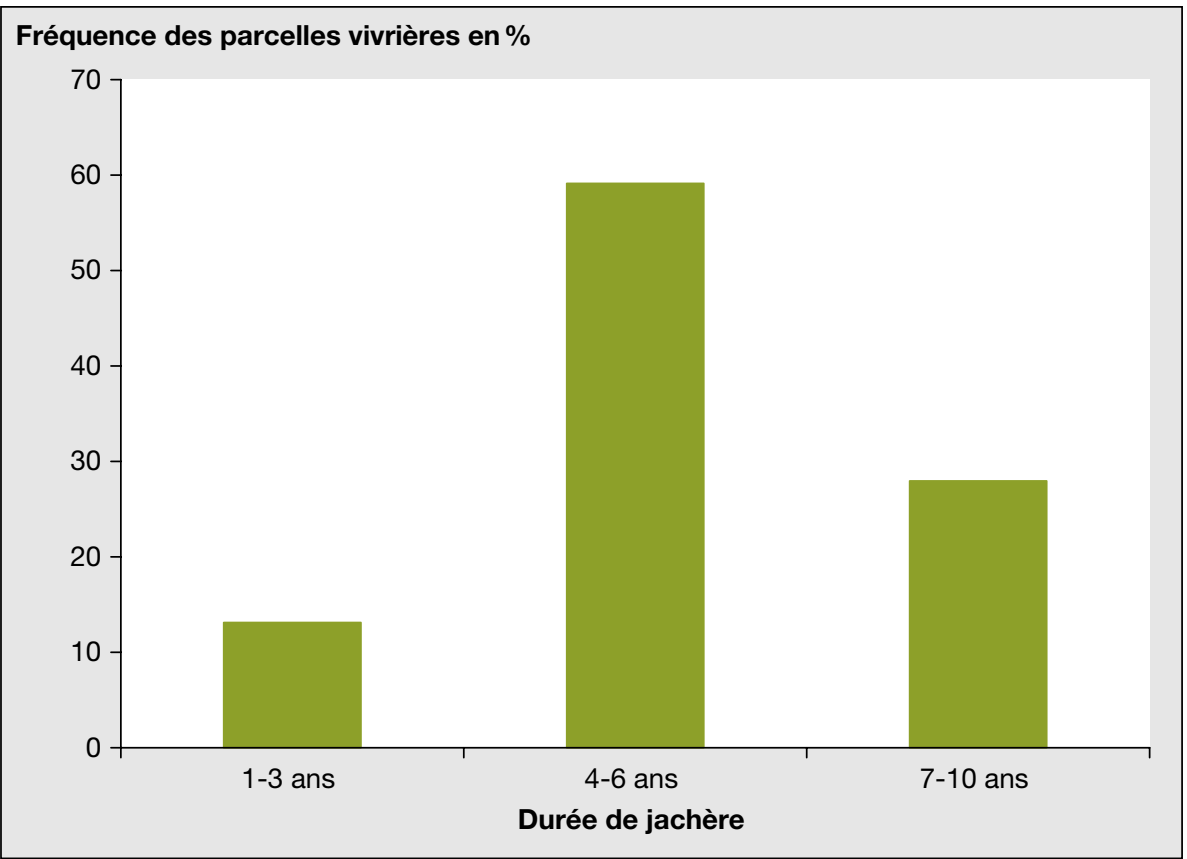

Figure 3. Variabilité de la durée de jachère pour les parcelles vivrières à base de riz sur coteaux (473 parcelles pour les trois villages d'étude en 2005).

Figure 3. Fallow duration variability for rice-based cropping systems on hills (473 plots in the three villages studied in 2005).

(tableau 2) permet d'identifier des éléments de compréhension des dynamiques de plantation dans cette région.

- les jeunes agroforêts de moins de 6 ans (encore improductives), représentent $31 \%$ des agroforêts. Il y a bien une dynamique récente et toujours actuelle de plantation qui s'accompagne d'une diversification des cultures pérennes, car le caféier et le kolatier très présents dans les vieilles agroforêts n'occupent plus que $25 \%$ des jeunes agroforêts. Spatialement ces extensions se structurent de la façon suivante :

- les jeunes agroforêts situées à moins d'un kilomètre du village sont des replantations ou des densifications d'anciennes agroforêts de la ceinture périvillageoise ;

- la couronne 1 à 3 kilomètres du village concentre la moitié des jeunes agroforêts qui constituent l'extension de la ceinture agroforestière périvillageoise.

Au-delà de cette ceinture, de 3 à 12 kilomètres du village, les nouvelles agroforêts sont plus rares $(30 \%)$ et se développent le long des axes de communication. Ces agroforêts proviennent parfois des anciens jardins de case de campement de culture déjà mentionnés. Elles correspondent aussi à des plantations sur des sites agropédologiques particuliers :

\section{Tableau 2. Âge et distance des agroforêts par rapport au centre du village.}

Table 2. Agroforest ages and distributions from the village centre.

\begin{tabular}{|c|c|c|c|c|c|c|c|c|}
\hline \multirow[t]{2}{*}{ Âge des agroforêts } & \multirow{2}{*}{$\begin{array}{c}\text { Nombre } \\
\text { de parcelles }\end{array}$} & \multirow[t]{2}{*}{$\%$} & \multicolumn{6}{|c|}{ Distance au village } \\
\hline & & & $\begin{array}{l}0-1 \\
\text { km }\end{array}$ & $\%$ & $\begin{array}{l}1-3 \\
\text { km }\end{array}$ & $\%$ & $\begin{array}{c}3-12 \\
\text { km }\end{array}$ & $\%$ \\
\hline 1 à 6 ans & 281 & 31 & 53 & 21 & 143 & 34 & 85 & 36 \\
\hline 7 à 30 ans & 541 & 60 & 166 & 67 & 241 & 58 & 134 & 57 \\
\hline 31 à 80 ans & 80 & 9 & 30 & 12 & 32 & 8 & 18 & 7 \\
\hline Total & 902 & 100 & 249 & 100 & 416 & 100 & 237 & 100 \\
\hline
\end{tabular}

anciens hameaux au sol riche, reliques de forêts, forêts galeries de bas-fonds. Cela correspond aussi au besoin de jeunes ménages qui cherchent à s'approprier de nouvelles terres propices aux cultures pérennes en s'éloignant du village.

- les agroforêts en production (7 à 30 ans) représentent $60 \%$ du total des agroforêts. Cela traduit bien la dynamique d'extension des agroforêts observée par la cartographie diachronique pour la période 1979-2003, suite à la libéralisation économique en Guinée datant de 1984. - les anciennes agroforêts (plus de 30 ans) ne représentent plus que $9 \%$ du total des agroforêts recensées en 2005 ; elles sont principalement situées dans la ceinture périvillageoise (moins de 3 kilomètres). Cela traduit l'absence de dynamique de plantation pendant la première République (1958-1984) caractérisée par un système de taxation, notamment du café, qui a dissuadé les paysans d'investir dans ce secteur (Rossi, 1993; Delarue, 2007). Ces analyses montrent qu'il y a eu ces dernières années une forte dynamique de plantation et de replantation dans ces trois villages, les superficies en agroforêts ayant presque doublé partout entre 1979 et 2003. Les raisons de cette dynamique sont-elles économiques et/ou patrimoniales? Quels en sont les effets sur la durabilité économique, écologique et sociale des systèmes de production?

\section{Pertinence et durabilité des systèmes de mise en valeur}

\section{Le système vivrier de coteaux : ancrage culturel et/ou performance économique?}

Selon Delarue (2007), la baisse du rendement en riz de coteaux est imputable à la réduction de la durée de la jachère précédente : 1 000-1 $200 \mathrm{~kg} / \mathrm{ha}$ après 10 ans de jachère versus $600-800 \mathrm{~kg} / \mathrm{ha}$ après une jachère de 4 ans environ. De ce fait, la productivité du travail diminue d'autant plus considérablement que la gestion des adventices dans un riz cultivé après 
une jachère de courte durée mobilise beaucoup plus de travail. Le maintien de ce type de riziculture n'est donc pas lié à un objectif simple de productivité économique. Il est sous-tendu aussi par des valeurs socioculturelles - nourrir sa famille avec son propre riz - et par des habitudes alimentaires liées aux qualités gustatives des variétés locales de riz. Néanmoins, une analyse uniquement centrée sur la production de riz sousestimerait la réelle performance économique des associations complexes "riz + autres vivriers + palmier à huile subspontané ". De fait, l' " huile rouge " artisanale issue de ces palmiers est devenue la première source de revenu monétaire des ménages ruraux de cette région. Il ne faut pas non plus négliger les productions des cultures associées souvent difficiles à quantifier et celles des cultures en rotation (arachide, manioc, banane) en années 2 et 3 après la défriche-brûlis. Ainsi, le manioc progresse partout et contribue de plus en plus à la sécurité alimentaire des ruraux. Mais la dégradation des conditions de production de ce système de culture est réelle: enherbement excessif surtout, et baisse de la fourniture de nutriments aux cultures par le sol. Ainsi, certains agriculteurs préfèrent abandonner la riziculture de coteaux pendant une année ou deux afin d'allonger la durée de la jachère, mais pour cela il leur faut disposer de rizières de bas-fonds pour assurer l'alimentation familiale. La réduction de la durée de jachère sera aussi dommageable aux palmiers à huile subspontanés si utiles aux familles. Du fait de l'invasion des jachères de courte durée par Chromolaena odorata, le recru forestier à base d'arbres héliophiles est compromis par le fort ombrage produit par cette espèce semi-ligneuse. De plus, son excellent rendement calorique lors du brulis précédent la remise en culture affecte la survie des jeunes palmiers (Gauthier, 1996). Dans ces conditions, le nombre de palmiers risque de décroître au fils des années.

\section{Les agroforêts : un système garant d'une certaine durabilité écologique}

L'association des cultures pérennes avec des espèces arborées forestières ne permet certainement pas d'espérer un rendement élevé de la culture principale. Son intérêt réside dans la diversification des productions et des revenus. Par ailleurs, l'effet des agroforêts en termes de résilience écologique est certainement important mais il n'a pas encore été quantifié. Les services environnementaux fournis par les agroforêts rappellent ceux d'une forêt secondaire (Griffon et Mallet, 1999) : réduction du ruissellement et de l'érosion - notamment pour les zones pentues et les berges des cours d'eau, entretien d'un microclimat plus humide, habitat pour la faune et surtout pour les auxiliaires qui limitent l'incidence des parasites des cultures. De plus, la protection et l'entretien d'espèces végétales dans ces agroforêts sont garants du maintien de la biodiversité et des usages multiples qui en découlent : bois d'œuvre, vannerie, pharmacopée, etc. Ces agroforêts constituent aussi un patrimoine qu'on peut léguer à ses descendants. Les agriculteurs reconnaissent deux avantages majeurs à ces agroforêts. Elles procurent avant tout une diversité de produits facilement commercialisables (café, noix de cola, cacao, bois, fruits divers, écorces) sans beaucoup de risques car l'investissement en travail et surtout en intrants est limité. Mais une des raisons majeures de l'intérêt porté aux agroforêts est le besoin pour les familles possédant beaucoup de terre de marquer leur emprise sur le foncier à une période où les demandes d'accession à la terre des jeunes ménages sont de plus en plus pressantes. Les qualités écologiques et les performances économiques de ce système de culture constituent aussi sur le long terme des éléments importants de la durabilité des systèmes de production de cette région.

\section{Mise en culture des bas-fonds : une intensification non maîtrisée}

En réponse à la réduction du temps de jachère et à la baisse des rendements du riz de coteaux, les bas-fonds sont aujourd'hui tous mis en valeur. La riziculture y est conduite le plus souvent en culture continue sans jachère et après un aménagement sommaire du bas-fond avec des diguettes en terre. La valorisation des bas-fonds en saison sèche reste encore modeste malgré une nappe phréatique très proche : un peu de niébé dérobé et quelques cultures maraîchères.

À partir des années 1990, l'État avec l'appui de bailleurs de fonds, a réalisé des aménagements hydro-agricoles des bas- fonds afin d'accroître la production du riz. L'objectif était, comme à Madagascar (Raunet, 1993) et au Vietnam (Erout et Castella, 2004) d'y produire une grande partie des besoins en riz des familles afin de réduire la riziculture pluviale sur coteaux et ainsi de préserver les dernières forêts. Mais dans la CRD de Kobela, les bas-fonds ne représentent qu'environ $5 \%$ des terres cultivables, trop peu pour constituer une alternative à la riziculture pluviale de coteaux. Par ailleurs, les différents aménagements se sont généralement révélés non durables après trois ou quatre années d'utilisation. La baisse rapide des rendements en riz dans ces situations a été observée et pourrait s'expliquer par le faible entretien des aménagements par les paysans et surtout la modification du fonctionnement hydrologique des bas-fonds: perturbation du régime hydrique des sols semitourbeux, isolement des bas-fonds aménagés de la sédimentation qui les alimentaient en éléments nutritifs (Delarue, 2007). Dans certains cas, des bas-fonds aménagés ne sont plus cultivés. La durabilité de la riziculture de bas-fonds aménagés et la rentabilité de cet investissement sont donc à tout le moins incertaines.

\section{Agencement spatial des différents systèmes de mise en valeur}

Chacun des systèmes de mise en valeur étudiés montre une certaine rationalité des producteurs. L'extension des agroforêts observée dans la CRD de Kobéla pose la question de la recherche de la meilleure combinaison entre les différents systèmes de culture sur les coteaux : riz pluvial + vivriers + palmier, d'une part, et agroforêts, d'autre part. Si l'extension des agroforêts se poursuit et correspond d'abord à une logique foncière, c'est la durabilité sociale du système agraire qui sera mise à mal : les familles détenant la terre posséderont de grandes superficies d'agroforêts et pourront de moins en moins prêter ou louer de la terre pour les cultures annuelles (riz, manioc...) aux familles qui possédaient à l'origine peu de terre. Par ailleurs, l'accroissement des surfaces d'agroforêts réduit d'autant plus la superficie disponible pour le système "riz de coteaux + vivriers " grand consommateur d'espace, déjà mis à mal par la réduction du temps de jachère. L'analyse de la durabilité des systèmes de production s'avère 
donc complexe et ne peut pas se limiter à la juxtaposition des analyses relatives à chaque système de culture. C'est bien à l'échelle du territoire villageois et à celui de l'exploitation agricole qu'il faut apprécier cette durabilité en tenant compte, par exemple, de la marginalisation de certains groupes d'agriculteurs ou de l'impact du développement d'un système de culture au détriment d'un autre sur la sécurité alimentaire.

\section{Conclusion}

Cette étude montre l'intérêt de l'approche géoagronomique pour comprendre les dynamiques spatiales des agrosystèmes et l'évolution des pratiques et des stratégies des agriculteurs. Cette démarche a permis aussi de discuter de la durabilité des systèmes de production qui en découlent. En Guinée forestière, ces 20 dernières années ont été marquées par une forte extension des agroforêts à base d'associations de cultures pérennes, s'appuyant sur les ceintures périvillageoises agroforestières anciennes. Cette dynamique spatiale des systèmes agricoles peut être assimilée à un cycle de renouvellement de l'écosystème initialement dominé par la forêt et les jachères de longue durée. Cette dynamique de reconstitution d'une "écologie forestière "n'est pas spécifique à la Guinée forestière. On l'observe aussi dans des zones moyennement à faiblement peuplées du Centre Cameroun où les agriculteurs développent les agroforêts à base de cacaoyers et de caféiers dans un écosystème de transition forêt-savane (Filipski et al., 2007). Dans les mêmes conditions climatiques, au nord de la Guinée forestière, Fairhead et Leach (1996) observent des pratiques paysannes de reforestation de la savane. Ainsi, les agriculteurs innovent en associant diverses espèces pérennes cultivées à des plantes subspontanées issues des écosystèmes initiaux. Ils confortent ainsi une stratégie de recherche d'un revenu sur le long terme en limitant les risques et en marquant leur emprise sur un foncier agricole de plus en plus convoité. Cette "reforestation ", que l'on devrait plutôt nommer "agroforestation ", ou "arborisation ", apporte des services environnementaux utiles à la collectivité (contrôle de l'érosion, maintien de la biodiversité, production de bois d'œuvre) et aboutit à des systèmes de culture montrant une bonne capacité de résilience écologique. Mais elle pourrait poser des problèmes d'installation des jeunes ménages et d'externalisation de la production vivrière en réduisant l'espace localement dévolu aux cultures vivrières. En cette période de hausse des prix des produits alimentaires de base (riz, huile, blé), il est important que les agronomes aident les agriculteurs à sécuriser leur production vivrière. L'enjeu pour la recherche et le développement est donc d'associer à la compréhension des stratégies paysannes, des démarches d'accompagnement de l'innovation et de prospective, afin de permettre à ces sociétés rurales d'atteindre leurs objectifs sans compromettre leur cohésion et leur avenir.

\section{Références}

Brasseur G. Études agricoles et économiques de quatre villages de Guinée Française, Guinée Forestière: Village de Niehen. Dakar (Sénégal) : Institut français d'Afrique noire, 1956.

Camara A. Dynamiques régionales et systèmes ruraux en Guinée forestière. Vers la conception d'un observatoire pour le développement. Thèse de géographie, Université d'Avignon et des pays de Vaucluse, 2007.
Deffontaines JP. Les sentiers d'un géoagronome. Paris: Editions Arguments, 1998.

Delarue J. Mise au point d'une méthode d'évaluation systémique de l'impact des projets de développement agricole sur le revenu des producteurs. Étude de cas en région kpèlè (République de Guinée). Thèse de doctorat en agriculture comparée, AgroParisTech, Paris, 2007.

Erout A, Castella JC. Riz d'en bas, riz d'en haut: éléments structurants des systèmes de production agricole d'une province de montagne du nord du Vietnam. Cah Agric 2004; $13: 413-20$.

Fairhead J, Leach M. Enriching the landscape: social history and the management of transition ecology in the forest savanna mosaic of the republic of Guinea. Africa : Journal of the International African Institute 1996 ; 66 : 14-36.

Filipski M, Colin JP, Seignobos C. Émergence et évolution des droits de propriété dans un contexte d'abondance foncière. Le cas du pays Yambassa (Cameroun). Cah Agric 2007; 16 : 387-93. doi : 10.1684/agr.2007.0129

Gauthier L. Emprise des brousses à Chromolaena odorata sur le fond du V-baoulé (Côte d'Ivoire centrale). Journal d'Agriculture Traditionnelle et de Botanique Appliquée 1996; 39 : 307-21.

Griffon M, Mallet B. En quoi l'agroforesterie peut-elle contribuer à la révolution doublement verte? Bois For Trop 1999 ; 260 : 41-51.

Madelaine C, Malézieux E, Sibelet N, Manlay R. Semi-wild palm groves reveal agriculture change in Forest Guinea. Agroforestry Systems $2008 ; 73: 189-204$

Porteres R. Les noms des riz en Guinée. Paris : Laboratoire d'ethnobotanique du Muséum national d'histoire naturelle, 1966.

Raunet M. Bas-fonds et riziculture. Actes du séminaire d'Antananarivo, Madagascar, 9-14 décembre 1991. Collection colloques. Montpellier: Cirad-CA, 1993.

Rossi. G. Évolution politique, démographie et dynamique de I'environnement en Guinée forestière. Cahier d'Outre-Mer 1993; 46 : 253-72.

Ruf F. Booms et crises du cacao. Les vertiges de l'or brun. Économie et développement. Paris: Karthala, 1995. 